\title{
Meningococcal colonisation misdiagnosed as gonococcal pharyngeal infection
}

\author{
ROBERT C. NOBLE AND ROBERT M. COOPER* \\ From the Division of Infectious Diseases, Department of Medicine, University of Kentucky College of \\ Medicine, Lexington, Kentucky, USA
}

SUMMARY Three cases of pharyngeal gonorrhoea were misdiagnosed in patients harbouring meningococci which failed to utilise maltose in CTA medium. The microbiological identity of the isolates was established by other means. Although meningococci giving atypical reactions to carbohydrate utilisation tests occur only rarely, the possibility of a misdiagnosis of gonorrhoea, and its social and legal repercussions, should be considered.

\section{Introduction}

Meningococci and gonococci usually infect the host at specific and different sites. This predilection for certain areas of the host, however, is not always present. Contrary to the usual experience, meningococci may infect urogenital and anal sites and gonococci may infect the pharynx and central nervous system (Holmes et al., 1971. Wiesner et al., 1973; Faur et al., 1975a; Noble et al., 1979). Pharyngeal infection by Neisseria gonorrhoeae is now recognised as another manifestation of gonococcal disease and these infections are more common in homosexual men, pregnant women, and other individuals practising oral genital sexual contact (Wiesner et al., 1973; Courman et al. 1974). Meningococci may also be found in the pharyngeal cultures of healthy individuals and patients attending venereal disease clinics (Weisner et al., 1973; Noble et al., 1979). Carbohydrate utilisation reactions are necessary to distinguish meningococci from gonococci since both organisms may be present in the pharynx. Unfortunately, these reactions are not always reliable and confusion between the two pathogens can occur. The following case reports show that a misdiagnosis of pharyngeal gonorrhoea is possible in patients who harbour meningococci with atypical carbohydrate utilisation reactions.

*Present affiliation: Department of Internal Medicine, Lexington Clinic, Lexington, Kentucky

Address for reprints: Dr Robert C. Noble, Division of Infectious Diseases, Department of Medicine, University of Kentucky College of Medicine, Lexington, Kentucky 40536, USA

Received for publication 7 February 1979

\section{Case reports}

CASE 1

A 21-year-old, white, married woman was admitted to the University of Kentucky Medical Center because of increasing dyspnoea. During the previous two weeks she had been exposed to a sister who had varicella. Six days before her admission the patient developed attacks of shivering and fever accompanied by vesicular lesions, which appeared initially on her chest. Three days before admission, she began to have a dry, hacking cough and shortness of breath.

On admission, she was dyspnoeic with a florid vesiculopustular rash covering her entire body. She had a fever of $40^{\circ} \mathrm{C}$; her respiration rate was 60 per minute, her blood pressure $110 / 60 \mathrm{~mm} \mathrm{Hg}$, and her pulse rate regular at 110 per minute. The laboratory values indicated hypoxia, a normal white blood cell count with a predominance of stab cells, and a low platelet count. The chest radiograph showed diffuse fluffy infiltrates affecting all lung fields. A pharyngeal culture, performed because she complained of a sore throat, initially gave a positive result for gonococci. A cervical culture gave a negative result for gonococci. Following penicillin therapy, a repeat pharyngeal culture result for gonococci was negative. She made an uneventful recovery. At the time of her illness, she had been living at home with her parents because her husband had been in prison for one year. She denied any extramarital sexual contacts.

CASE 2

A 19-year-old, white, single man was seen in the LexingtonFayette County Venereal Disease Clinic complaining of an urethral discharge, dysuria of three days' duration, and a sore throat. He had a thin, mucoid, urethral discharge and cervical lymph nodes which were enlarged bilaterally. His tonsils were enlarged, but there was no exudate. He denied orogenital sexual contact. Microscopical examination of the urethral exudate showed numerous polymorphonuclear 
leucocytes but no bacteria. Culture of the urethral exudate showed no growth. His pharyngeal culture result was initially reported as positive for gonococci. He was treated with oral tetracycline and a follow-up pharyngeal culture result was negative.

\section{CASE 3}

A 20-year-old, white, married man attended the LexingtonFayette County Venereal Disease Clinic complaining of a painful urethral discharge of three days' duration. He did not have a sore throat. Clinical examination was unremarkable except for the presence of a purulent urethral exudate. Microscopical examination of the exudate showed numerous polymorphonuclear leucocytes and many Gramnegative intracellular diplococci. A urethral culture showed the presence of oxidase-positive, Gram-negative diplococci. Unfortunately, this culture was lost and could not be further identified. A pharyngeal culture result was initially reported as positive for gonococci. He was treated with oral ampicillin and probenecid but failed to return for a followup culture.

\section{Materials and methods}

\section{MICROBIOLOGICAL METHODS}

The pharyngeal cultures were taken with cottontipped swabs, inoculated on to modified ThayerMartin medium containing vancomycin, colistin, nystatin, and trimethoprim (Martin and Lester, 1971), and incubated for 24 hours at $36^{\circ} \mathrm{C}$ in an atmosphere containing $5 \% \mathrm{CO}_{2}$. The isolates were initially identified by bacterial morphology, Gram stain, and oxidase reaction $(1 \%$ solution of $N$, $\mathrm{N}$-dimethyl-p-phenylenediamine monohydrochloride). The sugar utilisation reactions were initially performed in CTA medium (Baltimore Biologic Laboratory, Cockeysville, Maryland). The medium contained the test carbohydrates, dextrose, maltose, sucrose, fructose, and lactose, in 1\% con- centrations. In addition, the isolates were identified by the modified rapid fermentation procedure of Kellogg and Turner (Brown, 1974). Fluorescent antibody tests on the isolates were performed with Bacto-FA $N$. gonorrhoeae serum (Difco Laboratories, Detroit, Michigan). Meningococcal serogrouping was performed by the slide agglutination technique with antisera for serogroups $A, B, C, D, X, Y$, and $Z$. The isolates were stored at $-70^{\circ} \mathrm{C}$ in tryptic soy broth (Difco) in $13 \%$ glycerol.

\section{GONOCOCCAL AUXOTYPING}

Auxotyping by the method of Catlin was performed twice on each isolate (Catlin, 1973). Gonococci of a known auxotype were included as controls.

\section{ACRYLAMIDE GEL ELECTROPHORESIS}

Gel electrophoresis of the cell proteins of the isolates was performed as previously described (Noble and Schell, 1978). The intact micro-organisms were lyophilised, heated in lauryl sulphate with 2-mercaptoethanol, and the solubilised cell proteins were subjected to alkaline sodium dodecyl sulphate gel electrophoresis. The resulting electrophoresis patterns were scanned with a spectrophotometer.

\section{Results}

The results are given in the Table. An acid reaction was present in all tubes containing dextrose when the isolates were tested in CTA medium and by the rapid fermentation procedure. We found no reaction in the tubes containing maltose and CTA medium even though the isolates were repeatedly tested and incubated for several days. The State reference laboratory tested the isolates in tubes containing maltose and CTA medium, however, and found a

Table Results of microbiological tests on isolates from three patients

\begin{tabular}{|c|c|c|c|}
\hline \multirow[b]{2}{*}{ Determination } & \multicolumn{3}{|c|}{ Results for isolates from Cases } \\
\hline & 1 & 2 & 3 \\
\hline $\begin{array}{l}\text { Growth on modified Thayer-Martin medium } \\
\text { Oxidase reaction } \\
\text { Gram-negative diplococcus } \\
\text { Fluorescein-labelled antigonococcal serum } \\
\text { Meningococcal antiserum } \\
\text { Carbohydrate utilisation } \\
\text { Dextrose } \\
\text { Maltose } \\
\text { Lactose } \\
\text { Fructose } \\
\text { Sucrose } \\
\text { Growth on auxotyping media } \\
\text { Complete medium } \\
\text { Cystine-deficient medium }\end{array}$ & $\begin{array}{l}+ \\
+ \\
+ \\
- \\
\text { Group A } \\
\text { CTA*/RFPt } \\
+1+ \\
-1+ \\
-1- \\
-1- \\
-1- \\
+ \\
+\end{array}$ & $\begin{array}{l}+ \\
+ \\
+ \\
- \\
\text { Group Z } \\
\text { CTA/RFP } \\
+1+ \\
-1+ \\
-1- \\
-1- \\
-1- \\
+ \\
+\end{array}$ & $\begin{array}{l}+ \\
+ \\
+ \\
- \\
\text { Group Z } \\
\text { CTA/RFP } \\
+/+ \\
-1+ \\
-1- \\
-1- \\
-1- \\
+ \\
+\end{array}$ \\
\hline
\end{tabular}


neutral rather than an acid reaction. Thus both laboratories found atypical reactions in the CTA medium containing maltose. Isolates tested by the rapid fermentation procedure produced an acid reaction in the tubes containing dextrose and maltose.

A negative reaction occurred when the isolates were tested by the fluorescein labelled antigonococcal serum. Growth of the isolates on the gonococcal auxotyping media was characteristic of meningococci rather than of gonococci. Gonococci require cystine and meningococci do not. All three isolates were able to grow in the absence of cysteine. The protein electrophoresis patterns of the three isolates differed from those observed with gonococcal isolates.

\section{Discussion}

Carbohydrate utilisation tests are generally used to confirm an isolate as either $N$. gonorrhoeae or Neisseria meningitidis. These tests are generally performed in a supporting medium and CTA medium is one of the most commonly used for this purpose. In the present study, CTA medium was the only medium used by the routine laboratory for this purpose. CTA medium has, however, been associated with aberrant utilisation of carbohydrates by both gonococcal and meningococcal isolates. The reaction pertinent to this report is that certain meningococci may fail to utilise maltose and are thus mistaken for gonococci (Kingsbury, 1967; Hollis et al., 1970; Faur et al., 1975b). The failure of some meningococci to utilise maltose has been associated with resistance to sulphonamide and is due to the absence of either maltose permease or maltose phosphorylase activity (Kingsbury, 1967).

Although a number of alternative media for the carbohydrate tests have been suggested, none has gained universal acceptance. The rapid fermentation procedure was used successfully in our report, but its use also has problems. Incorrect results may occur if the maltose used in this procedure is not absolutely pure (Kellogg and Turner, 1973; Brown, 1974; Morse and Bartenstein, 1976). Kellogg and Turner (1973) found that only one of four lots of one commercial source of maltose was satisfactory. Morse and Bartenstein (1976) also experienced difficulty with their maltose source and used maltose supplied by Brown (see above). The commercially available fluorescein-labelled antigonococcal serum used in the present study has been shown to contain crossreactions with meningococcal isolates. These have varied from weak (Blenk et al., 1978) to strong enough to cause difficulty in distinguishing meningococci from gonococci (Hare, 1974). Pollock (1976) found that $5 \%$ of nongonococcal Neisseria gave positive results when tested with this reagent. Non-commercial immunofluorescent antigonococcal sera are also used to identify gonococci (Lind, 1975). Olcen et al. (1978), however, believe that immunofluorescent tests with unabsorbed conjugates cannot be recommended for confirmation of gonococci from extragenital sites and that absorbed conjugates are less suitable for routine use since their reactivity may drop rapidly after one month of storage. Although gonococcal auxotyping may be helpful in distinguishing gonococci from meningococci in problem cultures, it is not practical for routine diagnostic use because the media are difficult to prepare. Patterns of cell proteins on acrylamide gels have not been sufficiently evaluated for diagnostic purposes, and the gel electrophoresis technique used in our study provided evidence only that the isolates were not gonococci.

The appearance of meningococci that fail to utilise maltose is fortunately uncommon; the frequency has ranged from 0.5 to $5.7 \%$ of meningococcal isolates from clinical surveys (Hollis et al., 1970; Faur et al., 1975b). This was also our experience. Cases 2 and 3 came to light as a result of pharyngeal cultures taken from 2000 patients attending a venereal disease clinic (Noble et al., 1979). Gonococcal colonisation of the pharynx was present in $1.3 \%(27 / 2000)$ and meningococcal colonisation in $9 \cdot 5 \%(189 / 2000)$ of the patients.

The physicians of our three patients were unaware that the carbohydrate utilisation reactions might be unreliable. The two isolates from the young men were first suspected of not being gonococci when their protein electrophoresis patterns were examined. The young woman was insistent that her diagnosis was in error, however, since she had been faithful to her husband, who at the time of her illness was in prison. Her story and complaints were initially regarded with scepticism. Although she accepted the reason for the error, the misdiagnosis of a sexually transmitted disease might have been more painful for her and her physician. A misdiagnosis of gonorrhoea may have additional legal and social implications if the patient is a child. Positive gonococcal culture results may be found in children who are victims of sexual abuse. Meningococci also occur occasionally, however, in the pharyngeal cultures of children (Gold et al., 1978), and confusion of these two neisseriae is possible. Physicians should be aware that the carbohydrate utilisation reactions, usually considered as the basic criterion of the diagnosis of Neisseria, may on occasion be misleading. This knowledge should encourage an open dialogue between the physician and the diagnostic laboratory in an effort to prevent a microbiological misdiagnosis of gonorrhoea. 
We thank Philip G. Weiler, Commissioner of Health, Lexington-Fayette County Health Department for his co-operation. We are also grateful to Ms Norma J. Cambron and Dr George E. Kilgore, Microbiology Branch, Department for Human Resources, Commonwealth of Kentucky, for their help with the identification of the meningococci and the fluorescent antibody determinations. We thank Dr W. K. Harrell, Biologic Reagents Section, Center for Disease Control, Atlanta, Georgia, and Dr M. D. Moody, Burroughs Wellcome Company, Research Triangle Park, NC, for their donation of the meningococcal grouping antisera. We appreciate the suggestions of Dr Ernest Stolz, Department of Dermatology, Erasmus University, Rotterdam, The Netherlands. The study was made possible by a grant from Alexandrine and Alexander Sinsheimer Fund.

\section{References}

Blenk, H., Junge, W., and Blenk, B. (1978). Fluorescent antibody technique in identification of Neisseria gonorrhoeae-microcolonies grown on membrane filters. Zentralblatt fur Bakteriologie Hygiene (Orig. A), 240, 480-488.

Brown, W. J. (1974). Modification of the rapid fermentation test for Neisseria gonorrhoeae. Applied Microbiology, 27, 1027-1030.

Catlin, B. W. (1973). Nutritional profiles of Neisseria gonorrhoeae and Neisseria meningitidis and Neisseria lactamica in chemically defined media and the use of growth requirements for gonococcal typing. Journal of Infectious Diseases, 128, 178-194.

Courman, L. C., Levinson, M. E., Knight, R., Carrington, E. R., and Kaye, D. (1974). The high frequency of pharyngeal gonococcal infection in prenatal clinic population. Journal of the American Medical Association, 230, 568-570.

Faur, Y. C., Weisburd, M. H., and Wilson, M. E. (1975a). Isolation of Neisseria meningitidis from the genito-urinary tract and anal canal. Journal of Clinical Microbiology, 2, 178-182.

Faur, Y. C., Weisburd, M. H., and Wilson, M. E. (1975b). Carbohydrate fermentation plate medium for confirmation of Neisseria species. Journal of Clinical Microbiology, 1, 294-297.
Gold, R., Goldschneider, I., Lepow, M. L., Draper, T. F., and Randolph, M. (1978). Carriage of Neisseria meningitidis and Neisseria lactamica in infants and children. Journal of Infectious Diseases, 137, 112-121.

Hare, M. J. (1974). Comparative assessment of microbiological methods for diagnosis of gonorrhoea in women. British Journal of Venereal Diseases, 50, 437-441.

Hollis, D. G., Wiggins, G. L., Weaver, R. E., and Schubert, J. A. (1970). Current status of lactose-fermenting Neisseria. Annals of the New York Academy of Sciences, 174, 444-449.

Holmes, K. K., Counts, G. W., and Beaty, H. N. (1971). Disseminated gonococcal infection. Clinical review. Annals of Internal Medicine, 74, 979-993.

Kellogg, D. S. and Turner, E. M. (1973). Rapid fermentation confirmation of Neisseria gonorrhoeae. Applied Microbiology, 25, $550-552$.

Kingsbury, D. T. (1967). Relationship between sulfadiazine resistance and the failure to ferment maltose in Neisseria meningitidis. Journal of Bacteriology, 94, 557-561.

Lind, I. (1975). Methodologic aspects of routine procedures for identification of Neisseria gonorrhoeae by immunofluorescence. Annals of the New York Academy of Sciences, 254, 400-406.

Martin, J. E. and Lester, A. (1971). Transgrow a medium for transport and growth of Neisseria gonorrhoeae and Neisseria meningitidis. Health Services and Mental Health Administration Reports, 86, 30-33.

Morse, S. A. and Bartenstein, L. (1976). Adaptation of the Minitek system for the rapid identification of Neisseria gonorrhoeae. Journal of Clinical Microbiology, 3, 8-13.

Noble, R. C., Cooper, R. M., and Miller, B. R. (1979). Pharyngeal colonisation by Neisseria gonorrhoeae and Neisseria meningitidis in black and white patients attending a venereal disease clinic. British Journal of Venereal Diseases, 55, 14-19.

Noble, R. C. and Schell, S. C. (1978). Acrylamide gel electrophoresis of proteins of Neisseria gonorrhoeae as an epidemiologic tool. Infection and Immunity, 19, 178-186.

Olcen, P., Danielsson, D., and Kjellander, J. (1978). Laboratory identification of pathogenic Neisseria with special regard to atypical strains: An evaluation of sugar degradation, immunofluorescence and co-agglutination tests. Acta Pathologica Microbiologica Scandinavica, Section B, 86, 327-334.

Pollock, H. M. (1976). Evaluation of methods for the rapid identification of Neisseria gonorrhoeae in a routine clinical laboratory. Journal of Clinical Microbiology, 4, 19-21.

Wiesner, P. J., Tronca, E., Bonin, P., Pedersen, A. H. B., and Holmes, K. K. (1973). Clinical spectrum of pharyngeal gonococcal infection. New England Journal of Medicine, 288, 181-185. 